\title{
An Evanescent Light Wave Cannot Possess a Transverse Spin
}

\author{
Chunfang Li, Yunlong Zhang \\ Department of Physics, Shanghai University, Shanghai, China \\ Email: cfli@shu.edu.cn
}

How to cite this paper: Li, C.F. and Zhang, Y.L. (2019) An Evanescent Light Wave Cannot Possess a Transverse Spin. Journal of Modern Physics, 10, 459-465.

https://dx.doi.org/10.4236/jmp.2019.104031

Received: February 28, 2019

Accepted: March 19, 2019

Published: March 22, 2019

Copyright (C) 2019 by author(s) and Scientific Research Publishing Inc.

This work is licensed under the Creative Commons Attribution International License (CC BY 4.0).

http://creativecommons.org/licenses/by/4.0/

\begin{abstract}
It is pointed out that the evanescent light wave occurring at total reflection does not possess a transverse spin angular momentum as Bliokh, Bekshaev, and Nori claimed recently in (2014) Nature Communications, 5, 3300. This is not only because of the nonlocality of the photon spin but also because the evanescent wave is such a state whose angular momentum cannot be separated into spin and orbital parts.
\end{abstract}

\section{Keywords}

Nonexistence, Transverse Spin, Evanescent Light Wave

\section{Introduction}

It was once believed [1] [2] that separating the photon angular momentum into its spin and orbital parts is physically meaningless. However, since the seminal work of Allen et al. [3], theoretical identification of spin and orbital parts of photon angular momentum have drawn much attention [4-14]. In a recent publication, Bliokh, Bekshaev, and Nori [15] claimed that the evanescent light wave occurring at total reflection has a transverse spin, which is independent of the polarization. They arrived at that conclusion by resorting to the so-called local densities for the spin and orbital angular momentum (OAM), which were constructed in Ref. [11].

Bialynicki-Birula [12] showed that even though the total angular momentum of a light wave is local, after the splitting into spin and orbital parts, "the locality is lost". Furthermore, one of the authors [16] found that the spin of the photon can be derived from a set of two relativistic quantum equations for those states with respect to which the momentum operator is Hermitian. The nonlocality of the photon spin originates in the nonlocality of the photon itself that is expressed by the relativistic quantum constraint. On the basis of these discussions, we will demonstrate in this paper that the notion of transverse spin advanced in Ref. [15] is physically incorrect. 


\section{Description of Demonstration Method}

For clarity, let us first take a brief look at how Bliokh et al. came to their conclusion. Consider a monochromatic light wave of angular frequency $\omega$ in the air. The local spin density they defined reads

$$
\boldsymbol{s}=\psi^{\dagger} \hat{\boldsymbol{S}} \psi=\frac{\gamma}{2} \operatorname{Im}\left(\boldsymbol{E}^{*} \times \boldsymbol{E}+\boldsymbol{H}^{*} \times \boldsymbol{H}\right),
$$

where $\hat{\boldsymbol{S}}=\left(\begin{array}{cc}\hat{\boldsymbol{\Sigma}} & 0 \\ 0 & \hat{\boldsymbol{\Sigma}}\end{array}\right)$ is a vector matrix, $\left(\hat{\Sigma}_{k}\right)_{i j}=-i \epsilon_{i j k}$ with $\epsilon_{i j k}$ the Levi-Civitá pseudotensor, $\psi=\sqrt{\frac{\gamma}{2}}\left(\begin{array}{c}\boldsymbol{E} \\ \boldsymbol{H}\end{array}\right)$ is the spinor wavefunction with $\boldsymbol{E}$ and $\boldsymbol{H}$ the complex electric and magnetic vectors, respectively, the superscript " + " denotes the conjugate transpose, the superscript " $*$ " denotes the complex conjugate, Gaussian units with $\gamma=(8 \pi \omega)^{-1}$ are used, and $\hbar=1$ is assumed. They claimed that the spin density is generated by the so-called spin part $\boldsymbol{p}^{S}$ of the momentum density in the following way,

$$
\boldsymbol{s}=\boldsymbol{x} \times \boldsymbol{p}^{S},
$$

where $\boldsymbol{x}$ is the position vector relative to the origin. Meanwhile, they claimed that $\boldsymbol{p}^{S}$ is expressed in terms of $s$ as

$$
\boldsymbol{p}^{S}=\frac{1}{2} \nabla \times \boldsymbol{s} .
$$

The local OAM density about the origin they defined is as follows,

$$
\boldsymbol{l}=\boldsymbol{x} \times \boldsymbol{p}^{O},
$$

where

$$
\boldsymbol{p}^{O}=\operatorname{Re}\left(\psi^{\dagger} \hat{\boldsymbol{P}} \psi\right)=\frac{\gamma}{2} \operatorname{Im}\left[(\nabla \boldsymbol{E}) \cdot \boldsymbol{E}^{*}+(\nabla \boldsymbol{H}) \cdot \boldsymbol{H}^{*}\right]
$$

is the so-called canonical part of the momentum density and $\hat{\boldsymbol{P}}=-i \nabla$ is the operator for the canonical momentum. They required that the sum of $\boldsymbol{p}^{S}$ and $\boldsymbol{p}^{O}$ should be equal to the momentum density that is proportional to the Poynting vector and is expressed in terms of the complex electric and magnetic vectors as

$$
\boldsymbol{p}=\gamma k_{0} \operatorname{Re}\left(\boldsymbol{E}^{*} \times \boldsymbol{H}\right),
$$

where $k_{0}=\omega / c$ is the wavenumber in the air. When applying Equation (1) to the evanescent wave that occurs at a total reflection, they found that $s$ is perpendicular to the propagation direction.

It is well known [17] [18] [19] that the angular momentum of a light wave about the origin is given by $\int \boldsymbol{x} \times \boldsymbol{p} d^{3} x$, where the integrand is known as the angular-momentum density about the origin,

$$
\boldsymbol{j}=\boldsymbol{x} \times \boldsymbol{p},
$$

and $\boldsymbol{p}$ is the momentum density (6). As a physically meaningful notion, the angular-momentum density has to be unique. So if $s$ in Equation (1) and $\boldsymbol{l}$ in Equation (4) are true local spin and OAM densities, respectively, one must have $\boldsymbol{s}+\boldsymbol{l}=\boldsymbol{j}$. This can also be seen from $\boldsymbol{p}^{S}+\boldsymbol{p}^{O}=\boldsymbol{p}$ if $\boldsymbol{s}$ is generated by $\boldsymbol{p}^{S}$ via Equation (2). To demonstrate the nonexistence of the transverse spin in an evanescent wave, it is enough to show that there is no such relation in that case. This is done below. 


\section{Demonstration of Nonexistence of the Transverse Spin}

Because it was claimed in Ref. [15] that the transverse spin in an evanescent wave is independent of the polarization, we consider such an evanescent wave that occurs when a TM-polarized plane wave is totally reflected by an interface between a dielectric medium of refractive index $n_{i}>1$ and the air of refractive index $n_{0}=1$ as is shown in Figure 1.

The complex magnetic vector of the evanescent wave in the air assumes the following form,

$$
\boldsymbol{H}=A \exp \left[i\left(\boldsymbol{k}_{0} \cdot \boldsymbol{x}-\omega t\right)\right] \bar{y}, \quad x \geq 0,
$$

where $A$ is a constant, $\boldsymbol{k}_{0}=i \kappa \bar{x}+k_{z} \bar{z}$ is the complex wavevector, $\kappa=\left(k_{z}^{2}-k_{0}^{2}\right)^{1 / 2}$ is the decay coefficient in the air, $k_{z}=k_{i} \sin \theta$, $k_{i}=n_{i} k_{0}, \theta$ is the incidence angle that is larger than the critical angle for total reflection $\theta_{c}=\sin ^{-1}\left(1 / n_{i}\right), \bar{x}, \bar{y}$, and $\bar{z}$ are unit vectors along the corresponding axes. The corresponding complex electric vector is given by

$$
\boldsymbol{E}=\frac{k_{z} \bar{x}-i \kappa \bar{z}}{k_{0}} A \exp \left[i\left(\boldsymbol{k}_{0} \cdot \boldsymbol{x}-\omega t\right)\right], \quad x \geq 0 .
$$

It is needless to say that the electric vector (9) is "perpendicular" to the associated complex wavevector, $\boldsymbol{E} \cdot \boldsymbol{k}_{0}=0$, as the Maxwell equation $\nabla \cdot \boldsymbol{E}=0$ requires. Substituting Equations (8) and (9) into Equation (6), we find for the momentum density,

$$
\boldsymbol{p}=\gamma k_{z}|A|^{2} \exp (-2 \kappa x) \bar{z}
$$

which is in the propagation direction, the $z$-axis. Upon substituting it into Equation (7), we have for the angular-momentum density about the origin,

$$
\boldsymbol{j}=\gamma k_{z}|A|^{2} \exp (-2 \kappa x)(y \bar{x}-x \bar{y}) .
$$

It is transverse, having both $x$ and $y$ components.

Substituting Equations (8) and (9) into Equation (1), one gets

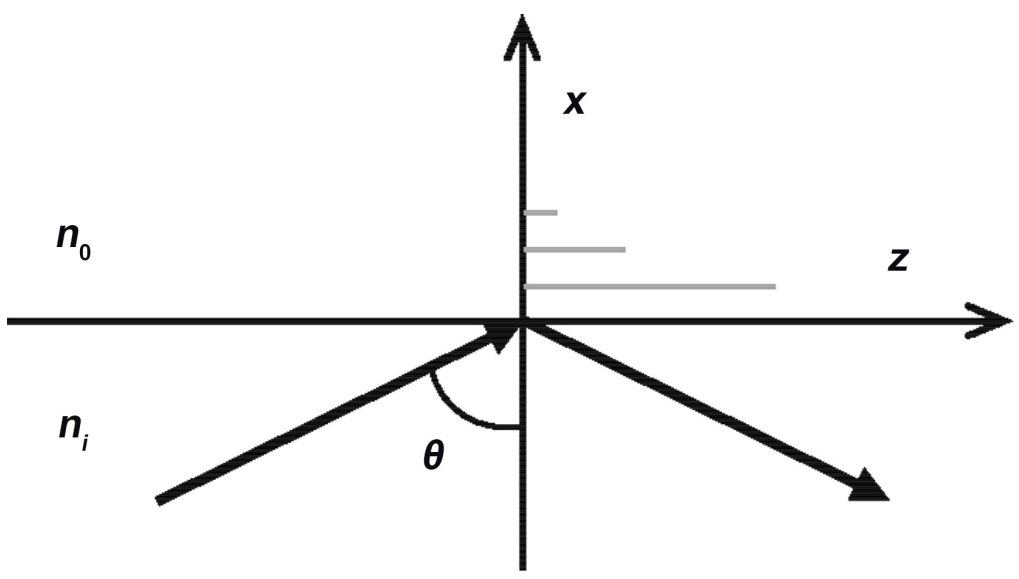

Figure 1. The evanescent wave that occurs at the total reflection of a TM-polarized plane wave by an interface at $x=0$ between two dielectric media of refractive indices $n_{i}$ and $n_{0}$. 


$$
\boldsymbol{s}=\frac{\gamma k_{z} \kappa}{k_{0}^{2}}|A|^{2} \exp (-2 \kappa x) \bar{y},
$$

which is in the transverse $y$ direction. It is on the basis of this result that Bliokh et al. [15] claimed that the evanescent wave possesses a transverse spin. However, substituting Equations (8) and (9) into Equation (5), one finds

$$
\boldsymbol{p}^{O}=\frac{\gamma k_{z}^{3}}{k_{0}^{2}}|A|^{2} \exp (-2 \kappa x) \bar{z},
$$

which, when substituted into Equation (4), gives

$$
\boldsymbol{l}=\frac{\gamma k_{z}^{3}}{k_{0}^{2}}|A|^{2} \exp (-2 \kappa x)(y \bar{x}-x \bar{y})
$$

Obviously, the sum of $\boldsymbol{s}$ and $\boldsymbol{l}$ in Equations (12) and (14) is different from the angular-momentum density (11). Instead, one has

$$
\boldsymbol{s}+\boldsymbol{l}=\frac{\gamma k_{z}}{k_{0}^{2}}|A|^{2} \exp (-2 \kappa x)\left[k_{z}^{2} y \bar{x}+\left(\kappa-k_{z}^{2} x\right) \bar{y}\right] .
$$

From this result it is concluded that the $\boldsymbol{s}$ in Equation (12) cannot be the local spin density and therefore the evanescent light wave does not possess a transverse spin.

Furthermore, substituting Equation (12) into Equation (3), one has

$$
\boldsymbol{p}^{S}=-\frac{\gamma k_{z} \kappa^{2}}{k_{0}^{2}}|A|^{2} \exp (-2 \kappa x) \bar{z} .
$$

The sum of (13) and (15) is indeed equal to the momentum density (10). But upon substituting Equation (15) into Equation (2), one cannot find Equation (12). Instead, one obtains

$$
\boldsymbol{x} \times \boldsymbol{p}^{S}=-\frac{\gamma k_{z} \kappa^{2}}{k_{0}^{2}}|A|^{2} \exp (-2 \kappa x)(y \bar{x}-x \bar{y}),
$$

which, in addition to a $y$-component, has a $x$-component. This is understandable. Resulting from Equation (1), the $\boldsymbol{s}$ in Equation (12) is supposed to be independent of the choice of a reference point. Nevertheless, coming from Equation (2), expression (16) must depend on the reference point, the origin. This further demonstrates that the $s$ in Equation (12) is not the local spin density. After all, as pointed out by Bialynicki-Birula [12], "when one splits the total angular momentum into its orbital part and the intrinsic part, locality cannot be preserved".

It is noted that according to the definition in quantum mechanics [20], the expectation value of the canonical momentum taken with respect to state $\psi$ is given by

$$
\langle\hat{\boldsymbol{P}}\rangle=\frac{\int \psi^{\dagger} \hat{\boldsymbol{P}} \psi d^{3} x}{\int \psi^{\dagger} \psi d^{3} x} .
$$

If the evanescent wave (8) and (9) can be regarded as a photon state, the resultant expectation value of the canonical momentum is complex, $\langle\hat{\boldsymbol{P}}\rangle=\boldsymbol{k}_{0}=i \kappa \bar{x}+k_{z} \bar{z}$. This shows that the evanescent wave is such a state with respect to which the canonical-momentum operator is not Hermitian. In other words, the canonical momentum of 
the photon in the evanescent wave is not an observable from the point of view of quantum mechanics. Accordingly, the corresponding OAM represented by the operator $\boldsymbol{x} \times \hat{\boldsymbol{P}}$ is not an observable, either. As a consequence, dividing the angular momentum of the photon in the evanescent wave into spin and orbital parts is physically impossible. This also explains why the angular momentum density $\boldsymbol{j}$ given by Equation (11) is different from the sum of $\boldsymbol{s}$ and $\boldsymbol{l}$ in Equations (12) and (14).

\section{Discussions and Conclusions}

On a final note, the photon in the evanescent wave (8) and (9) does have a transverse angular momentum about the origin. To see this in more detail, let us calculate the energy density of the evanescent wave,

$$
u=\frac{1}{16 \pi}\left(|\boldsymbol{E}|^{2}+|\boldsymbol{H}|^{2}\right)=\frac{k_{z}^{2}}{8 \pi k_{0}^{2}}|A|^{2} \exp (-2 \kappa x) .
$$

Considering that the energy of each photon is $\omega$, we adopt the technique used in Ref. [6] to find the momentum of a single photon per unit length along the propagation direction,

$$
\boldsymbol{P}=\omega \frac{\int_{x \geq 0} \boldsymbol{p} d x d y}{\int_{x \geq 0} u d x d y}=\frac{k_{0}^{2}}{k_{z}} \bar{z} .
$$

Because of $\frac{k_{0}}{k_{z}}<1$, its magnitude, $P=\frac{k_{0}^{2}}{k_{z}}$, is less than that of the momentum of free photon in the air, $k_{0}$. Correspondingly, from Equation (11) we have for the angular momentum of a single photon per unit length along the propagation direction,

$$
\boldsymbol{J}=\omega \frac{\int_{x \geq 0} \boldsymbol{j} d x d y}{\int_{x \geq 0} u d x d y}=-\frac{P}{2 \kappa} \bar{y},
$$

which is in the transverse $y$ direction. According to the definition of the angular momentum of a point particle about the origin, $\boldsymbol{J}=\boldsymbol{x} \times \boldsymbol{P}$, it follows from preceding two equations that the $x$-component of the photon's position vector $\boldsymbol{x}$ at any instant is $x=\frac{1}{2 \kappa}$ if we consider the photon as a point particle. That is to say, the photon behaves as a point particle of momentum $P$ along the propagation direction with a distance $\frac{1}{2 \kappa}$ away from the interface. The effect of such a transverse angular momentum was observed twenty years ago [21] in an experiment on combined Mie particles and was then explained as the result of the vertical gradient of the longitudinal radiation pressure that is expressed by the momentum density (10).

In conclusion, the evanescent light wave does not possess a transverse spin not only because of the nonlocality of the photon spin but also because the angular momentum of the evanescent wave cannot be separated into spin and orbital parts.

\section{Acknowledgements}

This work was supported in part by the program of Shanghai Municipal Science and Technology Commission (18ZR1415500).

\section{Conflicts of Interest}

The authors declare no competing financial interests. 


\section{References}

[1] Akhiezer, A.I. and Berestetskii, V.B. (1965) Quantum Electrodynamics. Interscience, New York.

[2] Cohen-Tannoudji, C., Dupont-Roc, J. and Grynberg, G. (1989) Photons and Atoms. John Wiley \& Sons, New York.

[3] Allen, L., Beijersbergen, M.W., Spreeuw, R.J.C. and Woerdman, J.P. (1992) Physical Review A, 45, 8185-8189. https://doi.org/10.1103/PhysRevA.45.8185

[4] van Enk, S.J. and Nienhuis, G. (1994) Europhysics Letters, 25, 497-501. https://doi.org/10.1209/0295-5075/25/7/004

[5] van Enk, S.J. and Nienhuis, G. (1994) Journal of Modern Optics, 41, 963-977. https://doi.org/10.1080/09500349414550911

[6] Barnett, S.M. and Allen, L. (1994) Optics Communications, 110, 670-678. https://doi.org/10.1016/0030-4018(94)90269-0

[7] Barnett, S.M. (2002) Journal of Optics B: Quantum and Semiclassical Optics, 4, S7-S16. https://doi.org/10.1088/1464-4266/4/2/361

[8] Barnett, S.M. (2010) Journal of Modern Optics, 57, 1339-1343. https://doi.org/10.1080/09500341003654427

[9] Li, C.-F. (2009) Physical Review A, 80, Article ID: 063814.

[10] Li, C.-F. (2016) Physical Review A, 93, Article ID: 049902(E).

[11] Bliokh, K.Y., Dressel, J. and Nori, F. (2014) New Journal of Physics, 16, Article ID: 093037. https://doi.org/10.1088/1367-2630/16/9/093037

[12] Bialynicki-Birula, I. (2014) New Journal of Physics, 16, Article ID: 113056. https://doi.org/10.1088/1367-2630/16/11/113056

[13] Barnett, S.M., Allen, L., Cameron, R.P., Gilson, C.R., Padgett, M.J., Speirits, F.C. and Yao, A.M. (2016) Journal of Optics, 18, Article ID: 064004

[14] Leader, E. (2018) Physics Letters B, 779, 385-387. https://doi.org/10.1016/j.physletb.2018.02.029

[15] Bliokh, K.Y., Bekshaev, A.Y. and Nori, F. (2014) Nature Communications, 5, Article No. 3300. https://doi.org/10.1038/ncomms4300

[16] Li, C.-F. (2019) Deriving Photon Spin from Relativistic Quantum Equations: Relativistic Quantum Constraint and Nonlocality of Photon Spin. arXiv:1806.04540v2

[17] Stratton, J.A. (1941) Electromagnetic Theory. McGraw-Hill, New York.

[18] Mandel, L. and Wolf, E. (1995) Optical Coherence and Quantum Optics. Cambridge University Press, Cambridge. https://doi.org/10.1017/CBO9781139644105

[19] Jackson, J.D. (1999) Classical Electrodynamics. 3rd Edition. John Wiley \& Sons, New York. 
[20] Sakurai, J.J. (1985) Modern Quantum Mechanics. Benjamin/Cummings, San Francisco, CA.

[21] Song, Y.G., Chang, S. and Jo, J.H. (1999) Japanese Journal of Applied Physics, 38, L380-L383.

https://doi.org/10.1143/JJAP.38.L380 